\title{
Time-course of running treadmill adaptation in novice treadmill runners
}

\section{Laura Simoni, Guido Pasquini , Silvia Pancani , Federica Vannetti , Claudio} Macchi \& Silvia Pogliaghi

To cite this article: Laura Simoni, Guido Pasquini , Silvia Pancani , Federica Vannetti , Claudio Macchi \& Silvia Pogliaghi (2020): Time-course of running treadmill adaptation in novice treadmill runners, Journal of Sports Sciences, DOI: 10.1080/02640414.2020.1782567

To link to this article: https://doi.org/10.1080/02640414.2020.1782567

册Published online: 23 Jun 2020.

Submit your article to this journal $₫$

Q View related articles \ulcorner

View Crossmark data $[\pi$ 


\title{
Time-course of running treadmill adaptation in novice treadmill runners
}

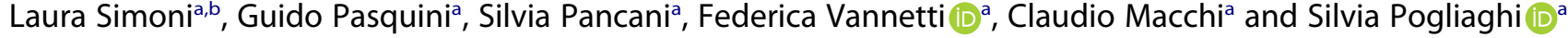 \\ ${ }^{a}$ Don Gnocchi Foundation IRCCS, Florence, Italy; ${ }^{b}$ Department of Neurosciences, Biomedicine and Movement Sciences, University of Verona, Verona, \\ Italy
}

\section{ABSTRACT}

Studies on running biomechanics and energetics are usually conducted on a treadmill. To ensure that locomotion on a treadmill is comparable to locomotion overground, participants need to be expert in the use of the device. This study aimed to identify the number and duration of sessions needed to obtain stable measurements for spatiotemporal and metabolic parameters in unexperienced treadmill runners. Fourteen male recreational runners performed three 15-min treadmill running trials in different days at a submaximal speed. Spatiotemporal and metabolic parameters were registered at minutes: 5, 10, 15 and their within-trial and between-trial changes were analysed using a two-way repeated measures ANOVA and Bonferroni post-hoc test. Within-trial differences were found in step frequency (decreased over time), Step Length and Contact Time (increased), reaching stability at different time points. Ventilator parameters increased, reaching stability after 5-10 $\mathrm{min}$, while heart rate increased progressively over time. The only between-trial differences were an increase in step length and a decrease in step frequency at min 1 , between trials 1 and 3 . In conclusion, at least three running trials of 15 min are required to familiarize with the device. The last $5 \mathrm{~min}$ of the third trial can be regarded as stable measurements.
ARTICLE HISTORY

Accepted 2 June 2020

KEYWORDS

Treadmill; treadmill running; adaptation; habituation; familiarization

\section{Introduction}

The use of treadmill offers many advantages in studying locomotion gait compared to overground running. Speed, slope, environmental factors can be easily controlled over a wide number of gait cycles (Lavcanska et al., 2005). For this reason, studies on running kinematics and energetics usually take place on a treadmill (McNeill et al., 2015). Moreover, treadmill is widely used also in rehabilitation and coaching field because it permits to easily observe and retrain running patterns. The most diffused types of treadmill are motorised flat treadmill. In addition, non-motorised devices are also available either with a flat or a curved surface, that generate distinct dynamic biomechanics and energetic responses compared to motorised devices (Bruseghini et al., 2019; Montgomery et al., 2016). Despite the many advantages that treadmills provide, running on treadmill can be at the beginning an unfamiliar experience, cause to the narrow belt, the constant visual field, the differences in perception and, for motorised treadmills, the imposed velocity (Fu et al., 2015; Hong et al., 2012; McNeill et al., 2015; Sloot et al., 2014). Inexperience in the use of treadmill can so lead participants to modify their natural running gait and may affect the metabolic responses to exercise (Arnold et al., 2019; Lavcanska et al., 2005).

Hence, repeated practice may help in developing an adaptation to treadmill running (Ogawa et al., 2015). Adaptation includes a process of familiarization and a process of habituation: familiarization refers to the within-trial stabilization (shortterm process) of a specific running pattern (Matsas et al., 2000), while habituation refers to a between-trial stabilization (longterm process) (Wall \& Charteris, 1981).
Habituation, not only familiarization, is a necessary condition to ensure the validity of both a cross-sectional and a longitudinal running evaluation on the treadmill.

There are several aspects of running, both related to kinematics and energetics, that have been described to change over repeated treadmill practices; however, investigations usually focus on either the bioenergetic (e.g., VO2) or the kinematic aspects of habituation; finally, the exact timing and mechanisms of habituation remain to be fully clarified.

With regard to motorised flat treadmills, several studies describe a distinctive familiarization upon initial exposure with the treadmill running, which consists of an increased stride length and a reduced step frequency over time. These features make treadmill locomotion increasingly more similar to overground running as habituation proceeds (Schieb, 1986; van Ingen, 1980). However, most of those studies did not investigate the time/number of trials needed for these features of habituation to occur. Only Schieb noted that stride length and step frequency stabilized either after a minimum of three training trials, each lasting $15 \mathrm{~min}$, or after a combination of trials with an accumulated training duration of approximately $45 \mathrm{~min}$ (Arnold et al., 2019). On the contrary, other kinematic parameters like contact and flight time seem not to be influenced by the habituation process (Schieb, 1986).

The energetic aspects of habituation to treadmill running have been scarcely evaluated (Morgan et al., 1994, 1991; Takabayashi et al., 2017). Since the metabolic response to running is influenced by spatiotemporal parameters such as stride length and step frequency, it is plausible that the metabolic adaptation to treadmill running could show similar 
characteristics to kinematic adaptation (Hunter et al., 2017). The scant data on the variability of the energy cost of treadmill locomotion over time in runners of unspecified adaptation suggested that at least $45 \mathrm{~min}$ of treadmill practice are needed for stable measurements of $\mathrm{VO}_{2}$ (Brueckner et al., 1991; Morgan et al., 1994, 1991). However, these relatively dated findings have not been confirmed in a specifically selected population naïve to treadmill locomotion.

Therefore, we studied the extent and time course of changes of kinematic and energetic variables resulting from familiarisation and habituation to the most common type of running ergometer, i.e., motorised flat treadmill. On the basis of the previous cited studies, we hypothesise that both the kinematic and energetic variables stabilise after a minimum of $45 \mathrm{~min}$ of accumulated activity (Morgan et al., 1994, 1991; Schieb, 1986).

\section{Methods}

Fourteen male recreational runners were recruited to participate in the study. Participants' characteristics are reported in Table 1. Females were not included in the study because of the mechanical differences with males in running (Hunter et al., 2017). Inclusion criteria were at least 4 years of experience in half-marathon running and a minimum seasonal best on halfmarathon between $1 \mathrm{~h} 20^{\prime}$ and $2 \mathrm{~h}^{\prime} \mathrm{O}^{\prime}$. Exclusion criteria were treadmill running familiarisation in the last 5 years and the presence of any musculoskeletal disease or illness, in the last 3 months, that could have influenced the participant's ability to run.

This study was approved by the ethical committee of the Don Gnocchi Foundation and all methods were performed in accordance with relevant guidelines and regulations of the institution. Informed consent was obtained from all individual participants included in the study.

Each participant performed three running trials of $15^{\prime}$ in a week at a constant speed on a flat motorised treadmill (MTCClimb, Runner, Italy). The running speed of each participant corresponded to that of his seasonal best on the half-marathon. This workload was assumed to minimize the fatigue factor which has been shown to affect running mechanics (Hunter et al., 2017). The three running trials were performed, with a minimum of $24 \mathrm{~h}$ of recovery, each day at the same time of the day and wearing the same footwear. Participants were requested not to train in the previous $24 \mathrm{~h}$.

Participants were permitted to use the handrails if they felt unsafe; however, none of the participants ended up using them during the tests.

Running analysis was carried out using marker-less optical system (Optogait, Microgate, Bolzano) to measure

Table 1. Participant demographics.

\begin{tabular}{ll}
\hline Variables & Mean \pm SD \\
\hline Age (years) & $33,8(13,4)$ \\
Weight $(\mathrm{kg})$ & $70,5(6,1)$ \\
Height $(\mathrm{cm})$ & $178,1(5)$ \\
BMI & $22,2(1,7)$ \\
Running experience (years) & $7(3)$ \\
Runs/week & $4(1)$ \\
Km of run/week & $39(9)$ \\
Best performance on $21 \mathrm{~km}(\mathrm{~km} / \mathrm{h})$ & $12,1(1,0)$ \\
\hline
\end{tabular}

spatiotemporal running parameters and a portable ergospirometric device (Oxycon Mobile, Jaeger, Germany) for the metabolic ones. Averages, standard deviation and coefficient of variation of all data were calculated for the final $30 \mathrm{~s}$ of four time windows $(1,5,10$ and $15 \mathrm{~min})$, for each trial. With regard to spatiotemporal parameters, 30 " of running corresponded at approximately 80 steps.

The validity and reliability of Optogait system were assessed by previous studies (Bernal et al., 2016; M. Lee et al., 2014; M. M. Lee et al., 2014). This device is constituted by two couples of transmitting and receiving 1-metre bars placed on the sides of the treadmill tape, at the level of the contact surface, connected to a computer controlled by the researcher. For each step, the following spatiotemporal running parameters were recorded: contact time $(C T)$, flight time (FT), step length $(\mathrm{SL})$ and step frequency (SF) (García-Pinillos et al., 2019; RocheSeruendo et al., 2018). These parameters were defined as follows (Roche-Seruendo et al., 2018):

- CT (s) is the time from the initial foot contact with the ground to the final lift off the ground of the same foot.

- FT (s) is the time from toe-off to initial foot contact with the ground of consecutive footfalls.

- SL (cm): was defined as the distance between two consecutive initial foot contacts. This parameter is calculated by summating the geometrical distance between two consecutive initial foot contacts and the distance travelled by the treadmill tape during the flight time (i.e., treadmill speed $x$ FT).

- SF (steps/sec): number of ground contact events per second.

Optogait software gives the possibility to increase its reliability by modifying the filter setting for the identification of gait events. Default setting of these parameters is often set up on 0_0 (i.e., CT begins when at least 1 LED is activated and finished once the number of LEDs activated returned to 0$)$, but other setting configurations can be selected to improve accuracy (1_1, 2_2, $\left.3 \_3 . ..\right)$. Based on pilot testing with concurrent video recording and in agreement with previous work in a similar setup (M. Lee et al., 2014) we chose to treat our data with a 1_1 filter setting.

The Oxycon Mobile system was used to measure metabolic parameters as previously described (Pasquini et al. 2015). In brief, runners were connected, through a mouthpiece and a low dead space valve, to the Oxycon Mobile's circuit system, which was calibrated before each test with gases of known concentration, according to the manufacturer's instructions. Oxygen uptake $\left(\mathrm{VO}_{2}, \mathrm{ml} / \mathrm{min}\right)$, respiratory exchange ratio (RER), ventilation (VE) and heart rate $(H R)$ were recorded using the instrument's rolling average filtering (i.e., on 8 breaths and the automatic discarding of the highest and lowest value of the 8). Data were exported as 5-s averages and a 30-s average (i.e., 6 data points) corresponding to the final $30 \mathrm{~s}$ of each time window (rest, 1, 5, 10 and $15 \mathrm{~min}$ ) was calculated. Metabolic data were also expressed as net units by subtracting rest values.

\section{Statistical analysis}

Normality of the data was checked by visual inspection of the data and by using the Shapiro-Whilk test. Data resulted normally distributed. A two-way analysis of variance (ANOVA) with 
repeated measures (trial number $\times$ time) was used to analyse the change in running parameters across different trials and different time points, to detect possible significant influence of time and/or number of trial on running mechanisms. When a significant main effect was found, pairwise post-hoc comparisons were performed to ascertain which trials/time points were significantly different from one another. To study familiarization process (influence of time) it was observed which time points did not show significant within-day differences with the min 15; habituation (influence of number of trials) was verified if there were no significant between-day differences in time points (Roche-Seruendo et al., 2018). Statistical differences were deemed as significant when $p<0.05$. Effect-size was calculated using partial-eta square (n2) (Cohen, 1973). A small effect was considered if values were $\geq 0.01$ and $<$ of 0.06 , medium if $>0$ of 0.06 and $<0.14$ and large if $\geq$ of 0.14 (Cohen, 1973).

Data were analysed using SPSS 21.0 (IBM SPSS Statistics, Chicago, IL).

\section{Results}

The group average of the absolute metabolic parameters as a function of time is summarised in Figure 1. Spatiotemporal and metabolic parameters within and between trials are summarised in Figures 2 and 3. In Figure 3, the net values of the metabolic parameters are displayed, to emphasise the possible differences within and between trials, above the resting baseline. Significant within-trial differences were observed for spatiotemporal and metabolic parameters, with the exception of FT. An increase in the mean value of the SL and CT and a decrease in the mean value of SF were observed from min 1 onward, in each trial. Both SL and SF were significantly different at min 1 and 5 compared to min 15 in all trials. P values for SL were 0.002 for trial 1 (T1), 0.003 for trial 2 (T2) and 0.023 for trial 3 (T3). P values for SF were 0.004 for T1, 0.044 for T2 and 0.015 for T3. In addition, for both variables in T1 min 10 differed significantly by $\min 15$ ( $p=0.002$ for $S L$ and $p=0.019$ for step frequency) and in $\mathrm{T} 2$, $\min 15$ showed a significant increase in SL compared to $\min 10$ ( $p=0.006)$. Effect size was large for all variables $\left(S L \eta^{2}=0.552, S F \eta^{2}=0.560\right)$. CT showed lower values compared to $\min 15$ at $\min 1 \mathrm{~T} 1(\mathrm{p}=0.001)$, and at $\min 1$ and 5 in T2 $(p<0.001$ and $p=0.012)$. Effect size was large $\left(\eta^{2}=0.642\right)$. Coefficient of variation (CV) of all spatiotemporal parameters did not change significantly with time.

All the metabolic parameters were significantly lower at min 1 compared to $\min 15$, in all the trials ( $p<0.0001$ ). HR was significantly lower compared to values at min 15 at all the time point of each trial too, with the exception of T2 where min 5 and 10 did not show significant differences with min 15 . In T1 and T2, p of min 5 vs 15 was less of 0.001 and p of min 10 vs $15=0.008$. In addition to what observed between min 1 and 15 , $\mathrm{VO}_{2}$ was significantly lower at min 5 vs 15 in T3 $(p=0.001)$, and VE in all the three trials. Differences within-trial were also observed for the $\mathrm{CV}$ of $\mathrm{VO}_{2}$ : $\min 1$ was significantly different to min 15 in all the trials ( $p<0.0001)$. Effect size was large for all metabolic parameters in every trial $\left(\mathrm{VO}_{2} \eta^{2}=0.918, \mathrm{HR}\right.$ $\left.\eta^{2}=0.853, \operatorname{RER} \eta^{2}=0.927, \operatorname{VE~} \eta^{2}=0.940, \mathrm{CV} \mathrm{VO}_{2} \eta^{2}=0.923\right)$.
Significant between-trial differences were found in the spatiotemporal parameters, but not in the metabolic ones and in the $\mathrm{CV}$ of $\mathrm{VO}_{2}$. In fact, SL and SF were significantly different between day 1 and day 3 at min 1, being $2.7 \mathrm{~cm}$ shorter and 0.05 stride/s higher, respectively $(p=0.031$ and $p=0.04)$. Effect size was large for all variables $\left(S L \eta^{2}=0.169, S F \eta^{2}=0.158\right)$.

\section{Discussion}

The purpose of this study was to establish the extent and time course of the changes of kinematic and energetic variables of treadmill running resulting from familiarisation (i.e., short-term, within-trials adjustment) and habituation (i.e., longer term, between-trial adaptation). In particular, we aimed at identifying the minimum number and duration of trials needed to obtain stable measurements for all the running parameters in experienced runners, naïve to motorised flat treadmill locomotion. The main finding of the study was that at least three 15-min running trials are needed to obtain stable measurements of all spatiotemporal and metabolic parameters, even if not all of them were equally affected by within and between adaptation processes.

In agreement with previous work (Cavanagh \& Williams, 1982; Schieb, 1986), within-trial adaptation was observed for all the spatiotemporal parameters with the exception of FT. SF decreased while SL and CT increased over the first 5-10 min of the trials. A different number of trials were necessary to obtain stable measures (i.e., no difference between $\min 1, \min 5, \min$ 10 and $\min 15$ ) for the different parameters. Only one conditioning trial plus a 5-min duration were necessary to obtain a stable CT. Regarding SL and step frequency, the values measured in the first minute of the first trial were both markedly different from the values prevailing in the successive time windows and trials. This finding is in agreement with Van Ingen Schenau, who described an apprehension to treadmill running upon initial exposure, which resulted in a more cautioned running state with an initial shortened SL and a higher SF (Hong et al., 2012); the above finding suggests that at least $5 \mathrm{~min}$ is necessary to allow the initial within-trial adaptation to occur during treadmill locomotion. Thereafter, one conditioning trial and a 10-min duration were necessary to obtain a stable SF while two trials and a 10-min duration were necessary for SL adaptation.

Lavcanska and Arnold, in two different studies about withintrial adaptation to treadmill running of kinematic parameters, indicate a shorter time of training needed to reach stability in a single trial, comprise between only 6 and 8 min (Lavcanska et al., 2005; Montgomery et al., 2016). Such discordance in the time may be a result of the considerable variability in study methodologies and in the characteristics of the population studied.

In particular, factors such as the level of treadmill and running experience of participants, the sampling rate of the gait analysis systems used and the number of data collected during trials may be influential (Arnold et al., 2019; Cavanagh \& Williams, 1982; McNeill et al., 2015). Runners studied in Lavcanska are younger and more trained which may contribute to a faster familiarization to new running conditions (Lavcanska et al., 2005). Moreover, the number of steps analysed was barely 

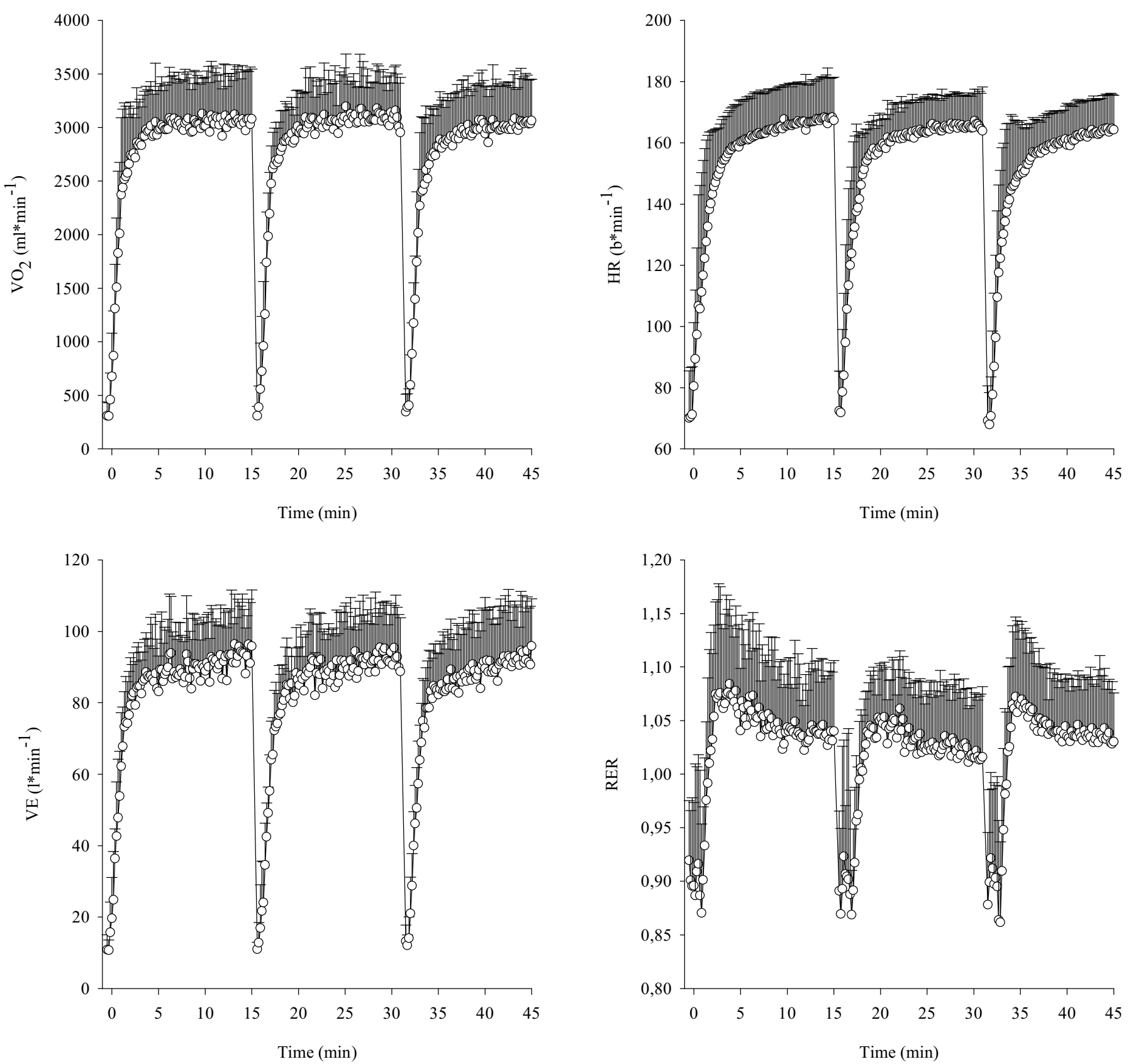

Figure 1. Row data of metabolic parameters during the three trials, as a function of time. The three trials conducted in different days have been appended and displayed in a single time line to facilitate direct comparison: $\min 1-15=$ trial $1 ; \min 15-30=$ trial $2 ; \min 30-45=$ trial 3.

at the limit of the recommendations (i.e., 10 steps vs 80 steps recorded in our study) while the sampling rate of the motion capture system was $60 \mathrm{~Hz}$ vs the currently recommended $150 \mathrm{~Hz}$. These features may have affected the accuracy and precision of measures and increased the likelihood of falsenegative results (i.e., earlier within-trial familiarisation due to lack of difference vs end-trial values).

Among the factors that could affect the pattern of adaptation to treadmill locomotion, our study did not directly measure vertical stiffness (Kvert) over time and trials. This parameter describes the vertical motion of the centre of mass during the contact phase and appears to be correlated to running performance, running economy and injury risk (Dutto \& Smith, 2002; Pappas et al., 2014; Taylor \& Beneke, 2012). Using the equation proposed by Morin et al. (Morin et al., 2005), we estimated the Kvert from CT, FT and the body mass of the participant. Based on these indirect estimates, the adaptation pattern of Kvert was similar to those seen for $\mathrm{CT}$, i.e., it was influenced only by within-trial time and not by the number of trials performed. Future studies should focus their attention also on this mechanical parameter and on the forces generated during running on the main segments of the lower body. This might give a more comprehensive understanding of the adaptive mechanism of biomechanical patterns during treadmill running.

As expected, the metabolic parameters displayed a delayed increase over time, that reflects the very well-known delayed adjustment of cardiorespiratory variables at the onset of a constant load exercise (Capelli et al., 2011). In agreement 

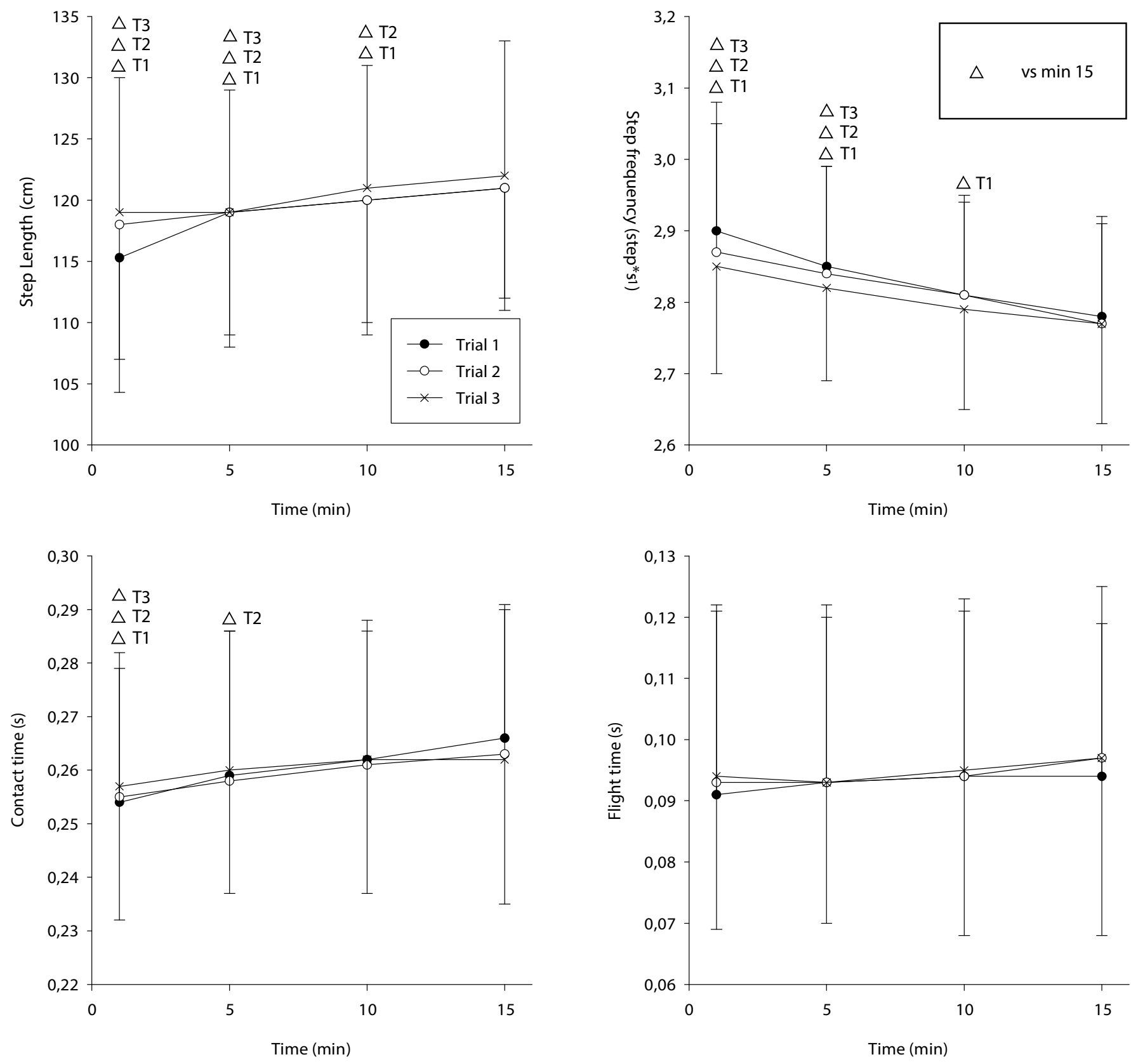

Figure 2. Significant differences between time and trials for spatio-temporal parameters. T1 - trial 1; T2 - trial 2; T3 - trial 3.

with previous studies from our group (Adami et al., 2011), stable measures were reached for ventilatory parameters within 5-10 min from exercise onset for all trials. On the contrary, HR continued to increase over time throughout the trials. This phenomenon is compatible with the appearance of the so-

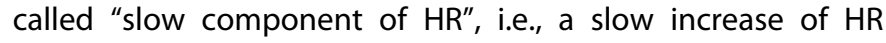
occurring at about 120-180 s into exercise, possibly also in absence of a slow component of $\mathrm{VO}_{2}$ (Zuccarelli et al., 2018). The lack of literature about the adaptation of metabolic parameters to treadmill running makes it difficult to compare the results of the current study with other researches. The few existing studies investigated the repeated practice of treadmill running in a sample of children or used a body-weightsupported treadmill (Cavanagh \& Williams, 1982; McNeill et al., 2015).
With regard to between-trial adaptation, except for the already mentioned fast adjustment of SF and SL during min 1 between $\mathrm{T} 1$ and $\mathrm{T} 3$, all the spatiotemporal parameters did not change at any time point between trials. Results are in agreement with Schieb et al., reporting that experienced over ground runners, new to treadmill, showed significant betweenday differences for SF and SL only in the very first minute of exercise (Schieb, 1986). Shortened and accelerated running gait is a strategy used to maintain stability when ambient perturbations, such as treadmill running for unaccustomed runners, take place (Latt et al., 2008; Lim et al., 2017; Schütte et al., 2016). Our study was the first to evaluate the within-participant variability of $\mathrm{VO}_{2}$ at 5, 10 and 15 min into exercise, over successive trials at identical speed. For the single speed evaluated in our study, $\mathrm{VO}_{2}$ at a given time point was highly consistent between trials 

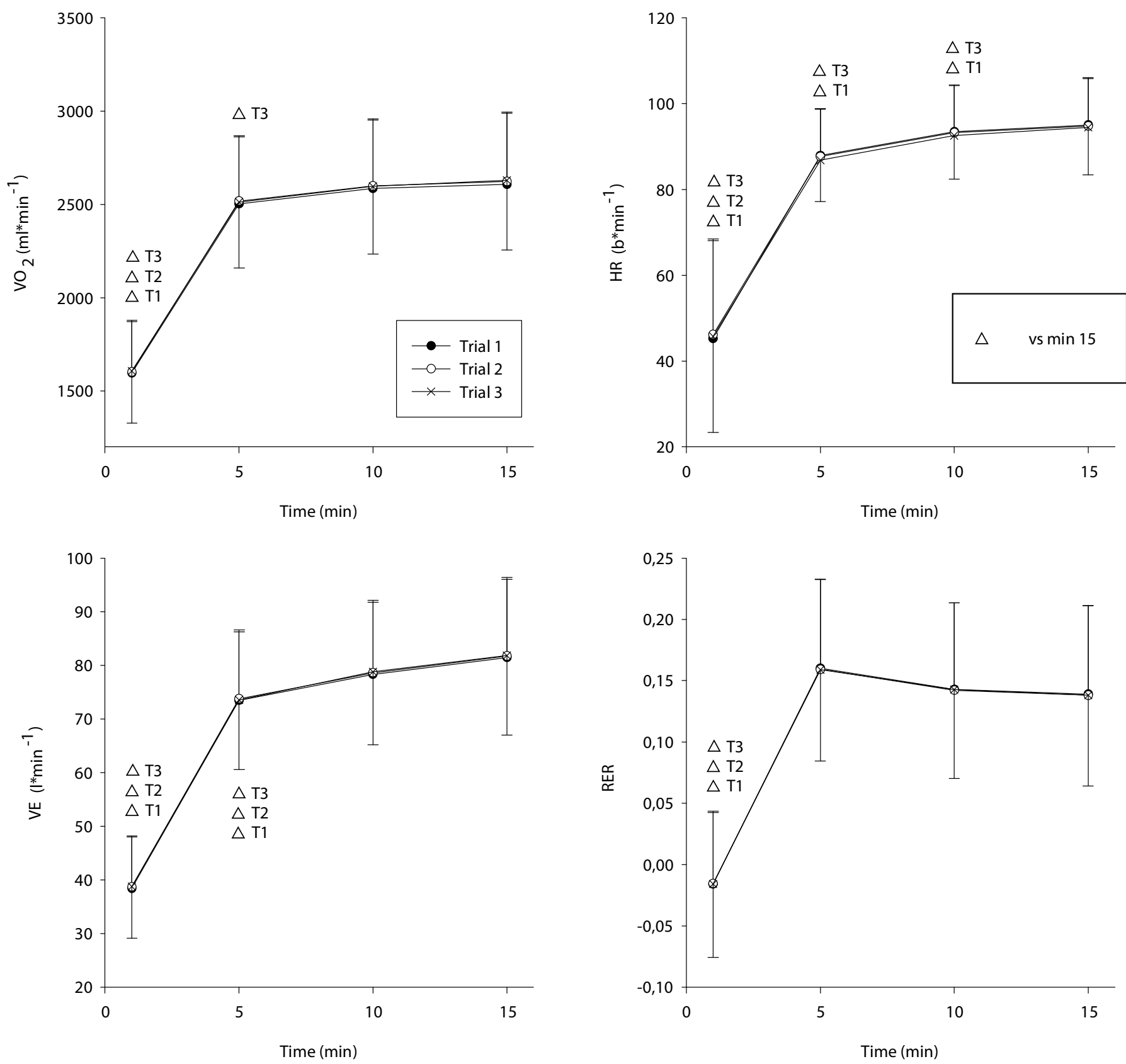

Figure 3. Significant differences between time and trials for metabolic parameters. $\mathrm{T} 1-$ trial $1 ; \mathrm{T} 2-$ trial $2 ; \mathrm{T} 3-$ trial 3.

(within-participant coefficient of variation equal to $0.047 \pm 0.004,0.050 \pm 0.006$ and $0.044 \pm 0.006$, respectively, at $\min 5, \min 10$ and $\min 15$ ). Therefore, contrary to our hypothesis, habituation showed by SL and SF between T1 and $\mathrm{T} 3$ at min 1 was not coupled with a decrease in $\mathrm{VO}_{2}$ between trials.

The above finding suggests the following: (i) the speed of adjustment of oxidative metabolism at exercise onset is unaffected by habituation to treadmill running; (ii) the amplitude of the habituation-induced changes in the kinematic parameters is not large enough to affect the energy cost of movement at the single speed evaluated in our study. Deviations of at least $\pm 8 \%$ from the preferred SL have been reported to increase oxygen consumption during submaximal running on treadmill (Brueckner et al., 1991). However, the between-trial difference in SL observed in our sample is not as large to determine significant differences in $\mathrm{VO}_{2}$, even if values at $\mathrm{T} 3$ are lower compared to $\mathrm{T} 1$ and $\mathrm{T} 2$. It may be hypothesized that a fourth trial could show an ulterior and significant decrease in metabolic measures.

Generalization of results about adaptation to treadmill running should be done with caution as the time and number of trials needed might be quite specific among different populations and running conditions (Cavanagh \& Williams, 1982; Schieb, 1986). Age, running experience, running velocity, type of shoes or barefoot conditions are factors supposed to influence adaptation time (Arnold et al., 2019; Lavcanska et al., 2005; McNeill et al., 2015; Ogawa et al., 2015; Schieb, 1986). Like suggested in previous studies, the adaptation process of inexperienced over ground runners would make an interesting 
successive study to this analysis of experienced over ground runners who were novice treadmill performers. The hypothesis is that inexperienced runners would probably exhibit a longer period of cautioned or apprehensive running (Agresta et al., 2019; Schieb, 1986). Furthermore, in spite of well-known differences in running responses depending on treadmill type (Bruseghini et al., 2019), there is a lack of information about running adaptation on either flat or curved, non-motorised devices. Future researches are needed to investigate the adaptation processes of biomechanical and energetics parameters on different treadmill types.

In conclusion, the current study has important practical implications. Usually, researches on running biomechanics and energetics are performed doing a few minutes of familiarization to treadmill before the test, but without any concern about the actual number of minutes needed to that specific population or the necessity to perform more than one training trial. In this study, it has been observed that familiarization and habituation processes affect spatiotemporal and metabolic parameters, in a different way depending on the considered variable. When studying run in recreational runners novice to motorised, flat treadmill, it is fundamental to perform at least three running trials of $15 \mathrm{~min}$ and to consider the last $5 \mathrm{~min}$. It cannot be excluded that in a fourth trial the running pattern may stabilize faster and that HR could reach a steady state too.

\section{Author contribution statement}

LS conceived and designed research, conducted experiments, analysed data and wrote the manuscript. GP designed research and conducted experiments. SP contributed with statistical expertise to analyse and interpret data and made a critical revision of the article for important intellectual content, FV conceived and designed research and made a critical revision of the article for important intellectual content, CM conceived and designed research, SP conceived and designed research, analysed data and wrote the manuscript. All authors read and approved the manuscript.

\section{Disclosure statement}

The authors report no conflict of interest

\section{ORCID}

Federica Vannetti (iD http://orcid.org/0000-0001-9403-7944

Silvia Pogliaghi (D) http://orcid.org/0000-0002-4394-8550

\section{References}

Adami, A., Pogliaghi, S., De Roia, G., \& Capelli, C. (2011). Oxygen uptake, cardiac output and muscle deoxygenation at the onset of moderate and supramaximal exercise in humans. European Journal of Applied Physiology, 111(7), 1517-1527. https://doi.org/10.1007/s00421-0101786-y

Agresta, C. E., Goulet, G. C., Peacock, J., Housner, J., Zernicke, R. F., \& Zendler, J. D. (2019). Years of running experience influences stride-tostride fluctuations and adaptive response during cadence perturbations in healthy distance runners. Gait \& Posture, 70, 376-382. https://doi.org/ 10.1016/j.gaitpost.2019.02.034

Arnold, B. J., Weeks, B. K., \& Horan, S. A. (2019). An examination of treadmill running familiarisation in barefoot and shod conditions in healthy men.
Journal of Sports Sciences, 37(1), 5-12. https://doi.org/10.1080/02640414. 2018.1479533

Bernal, A. G., Becerro-de-Bengoa-Vallejo, R., \& Losa-Iglesias, M. E. (2016). Reliability of the OptoGait portable photoelectric cell system for the quantification of spatial-temporal parameters of gait in young adults. Gait \& Posture, 50, 196-200. https://doi.org/10.1016/j.gaitpost.2016.08. 035

Brueckner, J. C., Atchou, G., Capelli, C., Duvallet, A., Barrault, D., Jousselin, E., Rieu, M., \& Di Prampero, P. E. (1991). The energy cost of running increases with the distance covered. European Journal of Applied Physiology and Occupational Physiology, 62(6), 385-389. https://doi.org/ 10.1007/BF00626607

Bruseghini, P., Tam, E., Monte, A., Capelli, C., \& Zamparo, P. (2019). Metabolic and kinematic responses while walking and running on a motorised and a curved non-motorised treadmill. Journal of Sports Sciences, 37(4), 396-403. https://doi.org/10.1080/02640414.2018. 1504605

Capelli, C., Cautero, M., \& Pogliaghi, S. (2011). Algorithms, modelling and VO2 kinetics. European Journal of Applied Physiology, 111(3), 343. https:// doi.org/10.1007/s00421-010-1396-8

Cavanagh, P. R., \& Williams, K. R. (1982). The effect of stride length variation on oxygen uptake during distance running. Medicine and Science in Sports and Exercise, 14(1), 30-35. https://doi.org/10.1249/00005768198201000-00006

Cohen, J. (1973). Eta-squared and partial eta-squared in fixed factor ANOVA designs. Educational and Psychological Measurement, 33(1), 107-112. https://doi.org/10.1177/001316447303300111

Dutto, D. J., \& Smith, G. A. (2002). Changes in spring-mass characteristics during treadmill running to exhaustion. Medicine and Science in Sports and Exercise, 34(8), 1324-1331. https://doi.org/10.1097/00005768200208000-00014

Fu, W., Fang, Y., Liu, D. M. S., Wang, L., Ren, S., \& Liu, Y. (2015). Surface effects on in-shoe plantar pressure and tibial impact during running. Journal of Sport and Health Science, 4(4), 384-390. https://doi.org/10.1016/j.jshs. 2015.09.001

García-Pinillos, F., García-Ramos, A., Ramírez-Campillo, R., LatorreRomán, P. Á., \& Roche-Seruendo, L. E. (2019). How do spatiotemporal parameters and lower-body stiffness change with increased running velocity? A comparison between novice and elite level runners. Journal of Human Kinetics, 70(1), 25-38. https://doi.org/10.2478/ hukin-2019-0036

Hong, Y., Wang, L., Li, J. X., \& Zhou, J. H. (2012). Comparison of plantar loads during treadmill and overground running. Journal of Science and Medicine in Sport, 15(6), 554-560. https://doi.org/10.1016/j.jsams.2012. 01.004

Hunter, I., Lee, K., Ward, J., \& TRACY, J. (2017). Self-optimization of stride length among experienced and inexperienced runners. International Journal of Exercise Science, 10(3), 446.

Latt, M. D., Menz, H. B., Fung, V. S., \& Lord, S. R. (2008). Walking speed, step frequency and step length are selected to optimize the stability of head and pelvis accelerations. Experimental Brain Research, 184(2), 201-209. https://doi.org/10.1007/s00221-007-1094-x

Lavcanska, V., Taylor, N. F., \& Schache, A. G. (2005). Familiarization to treadmill running in young unimpaired adults. Human Movement Science, 24 (4), 544-557. https://doi.org/10.1016/j.humov.2005.08.001

Lee, M., Song, C., Lee, K., Shin, D., \& Shin, S. (2014). Agreement between the spatio-temporal gait parameters from treadmill-based photoelectric cell and the instrumented treadmill system in healthy young adults and stroke patients. Medical Science Monitor: International Medical Journal of Experimental and Clinical Research, 20, 1210. https://doi.org/10.12659/ MSM.890658

Lee, M. M., song, C. H., Lee, K. J., Jung, S. W., Shin, D. C., \& Shin, S. H. (2014). Concurrent validity and test-retest reliability of the OPTOGait photoelectric cell system for the assessment of spatio-temporal parameters of the gait of young adults. Journal of Physical Therapy Science, 26(1), 81-85. https://doi.org/10.1589/jpts.26.81

Lim, J., Busa, M. A., Van Emmerik, R. E., \& Hamill, J. (2017). Adaptive changes in running kinematics as a function of head stability demands and their effect on shock transmission. Journal of Biomechanics, 52, 122-129. https://doi.org/10.1016/j.jbiomech.2016.12.020 
Matsas, A., Taylor, N., \& McBurney, H. (2000). Knee joint kinematics from familiarised treadmill walking can be generalised to overground walking in young unimpaired subjects. Gait \& Posture, 11(1), 46-53. https://doi. org/10.1016/S0966-6362(99)00048-X

McNeill, D. K., de Heer, H. D., Williams, C. P., \& Coast, J. R. (2015). Metabolic accommodation to running on a body weight-supported treadmill. European Journal of Applied Physiology, 115(5), 905-910. https://doi. org/10.1007/s00421-014-3071-y

Montgomery, G., Abt, G., Dobson, C., Smith, T., \& Ditroilo, M. (2016). Tibial impacts and muscle activation during walking, jogging and running when performed overground, and on motorised and non-motorised treadmills. Gait \& Posture, 49, 120-126. https://doi.org/10.1016/j.gaitpost.2016.06.037

Morgan, D. W., Craib, M. W., Krahenbuhl, G. S., Woodall, K., Jordan, S. Filarski, K., Burleson, C., \& Williams, T. (1994). Daily variability in running economy among well-trained male and female distance runners. Research Quarterly for Exercise and Sport, 65(1), 72-77. https://doi.org/ 10.1080/02701367.1994.10762210

Morgan, D. W., Martin, P. E., Krahenbuhl, G. S., \& Baldini, F. D. (1991) Variability in running economy and mechanics among trained male runners. Medicine and Science in Sports and Exercise, 23(3), 378-383. https://doi.org/10.1249/00005768-199103000-00018

Morin, J. B., Dalleau, G., Kyröläinen, H., Jeannin, T., \& Belli, A. (2005). A simple method for measuring stiffness during running. Journal of Applied Biomechanics, 21(2), 167-180. https://doi.org/10.1123/jab.21.2.167

Ogawa, T., Kawashima, N., Obata, H., Kanosue, K., \& Nakazawa, K. (2015). Distinct motor strategies underlying split-belt adaptation in human walking and running. PloS One, 10(3), e0121951. https://doi.org/10. 1371/journal.pone.0121951

Pappas, P., Paradisis, G., Tsolakis, C., Smirniotou, A., \& Morin, J. B. (2014). Reliabilities of leg and vertical stiffness during treadmill running. Sports Biomechanics, 13(4), 391-399. https://doi.org/10.1080/14763141.2014. 981853

Pasquini, G., Vannetti, F., \& Molino-Lova, R. (2015). Ability to work in anaerobic condition is associated with physical performance on the six-minute walk test in older patients receiving cardiac rehabilitation. Journal of Rehabilitation Medicine, 47(5), 472-477. https://doi.org/10. 2340/16501977-1956
Roche-Seruendo, L. E., García-Pinillos, F., Haicaguerre, J., Bataller-Cervero, A. V., Soto-Hermoso, V. M., \& Latorre-Román, P. Á. (2018). Lack of influence of muscular performance parameters on spatiotemporal adaptations with increased running velocity. The Journal of Strength \& Conditioning Research, 32(2), 409-415. https://doi.org/10.1519/JSC. 0000000000001845

Schieb, D. A. (1986). Kinematic accommodation of novice treadmill runners. Research Quarterly for Exercise and Sport, 57(1), 1-7. https://doi.org/10. 1080/02701367.1986.10605381

Schütte, K. H., Aeles, J., De Beéck, T. O., van der Zwaard, B. C., Venter, R., \& Vanwanseele, B. (2016). Surface effects on dynamic stability and loading during outdoor running using wireless trunk accelerometry. Gait \& Posture, 48, 220-225. https://doi.org/10.1016/j.gaitpost.2016. 05.017

Sloot, L. H., Van der Krogt, M. M., \& Harlaar, J. (2014). Effects of adding a virtual reality environment to different modes of treadmill walking. Gait \& Posture, 39(3), 939-945. https://doi.org/10.1016/j.gaitpost.2013. 12.005

Takabayashi, T., Edama, M., Nakamura, M., Nakamura, E., Inai, T., \& Kubo, M. (2017). Gender differences associated with rearfoot, midfoot, and forefoot kinematics during running. European Journal of Sport Science, 17(10), 1289-1296. https://doi.org/10.1080/17461391. 2017.1382578

Taylor, M. J. D., \& Beneke, R. (2012). Spring mass characteristics of the fastest men on Earth. International Journal of Sports Medicine, 33(8), 667-670. https://doi.org/10.1055/s-0032-1306283

van Ingen, S. G. (1980). Some fundamental aspects of the biomechanics of overground versus treadmill locomotion. Medicine and Science in Sports and Exercise, 12(4), 257

Wall, J. C., \& Charteris, J. (1981). A kinematic study of long-term habituation to treadmill walking. Ergonomics, 24(7), 531-542. https://doi.org/10. 1080/00140138108924874

Zuccarelli, L., Porcelli, S., Rasica, L., Marzorati, M., \& Grassi, B. (2018). Comparison between slow components of HR and V O2 kinetics: Functional significance. Medicine and Science in Sports and Exercise, 50(8), 1649-1657. https://doi.org/10.1249/MSS. 0000000000001612 